EPJ Web of Conferences 41, 04037 (2013)

DOI: $10.1051 /$ epjconf/20134104037

C) Owned by the authors, published by EDP Sciences, 2013

\title{
Hyperspectral Probing of Exciton dynamics and Multiplication in PbSe Nanocrystals
}

\author{
I. Gdor ${ }^{1}$, H. Sachs ${ }^{1}$, A. Roitblat ${ }^{1}$, D. Strasfeld ${ }^{2}$, M. G. Bawendi ${ }^{2}$ and S. Ruhman ${ }^{1}$ \\ ${ }^{1}$ Institute of Chemistry and the Farkas Center for Light Induced Processes, the Hebrew University, \\ Jerusalem 91904, Israel. \\ ${ }^{2}$ Department of Chemistry, Massachusetts Institute of Technology, 77 Massachusetts Avenue, \\ Cambridge, Massachusetts 02139, USA.
}

\begin{abstract}
Height time hyperspectral near IR probing providing broad-band coverage is employed on PbSe nanocrystals, uncovering spectral evolution following high energy photo-excitation due to hot exciton relaxation and recombination. Separation of single, double and triple exciton state contributions to these spectra is demonstrated, and the mechanisms underlying the course of spectral evolution are investigated. In addition no sign of MEG was detected in this sample up to a photon energy 3.7 times that of the band gap.
\end{abstract}

\section{Introduction.}

Exciton cooling and recombination in semiconductor Quantum Dots (QD) can be followed by Transient Absorption (TA) spectroscopy. TA has also served to search for Multi-Exciton Generation (MEG) where absorbance of one energetic photon generates multiple excitons in the same QD. Initial reports of highly efficient MEG in various QDs [1,2] has prompted hopes for economic solar energy conversion in QD based photovoltaics . Recently those reports have been questioned [3], stressing the need for detailed studies into exciton relaxation dynamics, such as that presented here.

Quantification of MEG efficiency has been based on exciton decay kinetics, where the presence of multiexcitons is revealed by their rapid decay due to Auger recombination. Inherent difficulties with this approach are; a) Multiple exciton states can also be generated by consecutive multiphoton absorption, and b) The pump fluences required for avoiding that becomes vanishingly small at high photon energies due to the steep exponential rise in QD cross section with reduction in excitation wavelength.

A pump-probe procedure for surmounting these difficulties was recently used to seek MEG in InAs core shell QDs[4]. It involves scaling both the sample, and the pump fluence, by $1 / \varepsilon\left(\lambda_{\text {pump }}\right)$. Direct comparison of exciton decay for equivalent experiments at two different excitation wavelengths can detect MEG since identical of exciton number states have been generated. Pump fluences needn't strictly avoid multiphoton excitation, and optically thick samples can be used, maximizing signal levels. No signs of MEG were detected in the InAs core-shell samples using this method up to a photon energy nearly 4 times the QD band gap [4]. Numerous reports of efficient $\mathrm{MEG}$, primarily in PbSe QDs have since been published. Here we aim to reapply the described method to that family of QDs as well, using an improved TA setup which provides continuous

This is an Open Access article distributed under the terms of the Creative Commons Attribution License 2.0, which permits unrestricted use, distribution, and reproduction in any medium, provided the original work is properly cited. 
hyperspectral probing, optimizing time and frequency resolution, and providing fine details of spectral evolution from which exciton dynamics can be inferred.

\section{Results and Discussion}

Transient difference spectrum recorded after photo-excitation with femtosecond pulses centered at $800 \mathrm{~nm}$ and $400 \mathrm{~nm}$ is presented in figure 1. The changes in probe transmission consist mainly of a sharp build-up of enhanced transmission centered near the initial absorption peaks, superimposed on a broad absorption which extends to longer wavelengths, but is apparent throughout. These features evolve within picoseconds as the nascent excitons cool. The changes observed consist mainly of a disappearance of the broad absorption background, leaving behind the excess transmission feature at the band edge, as well as a zero integral peak shift signature associated with the second absorption[5]. After this initial evolution, at much longer delay times, a gradual decay $(\sim 100 \mathrm{ps})$ is observed. This is the signature of exciton annihilation due to Auger recombination. Finally this leaves a long lived difference spectrum characteristic of single exciton, which does not decay significantly in our observation window.

Following the exciton cooling during the first few picoseconds reveals; 1) at both pump wavelengths, the entire transient spectrum seems at early delays to be dominated by a broad absorptive background extending throughout the probed range. 2) The apparent background is broader when exciting with the more energetic photons. 3) The amplitude of the localized bleaching component at the band edge is nearly conserved during the process of cooling independent of the exciting photon energy.

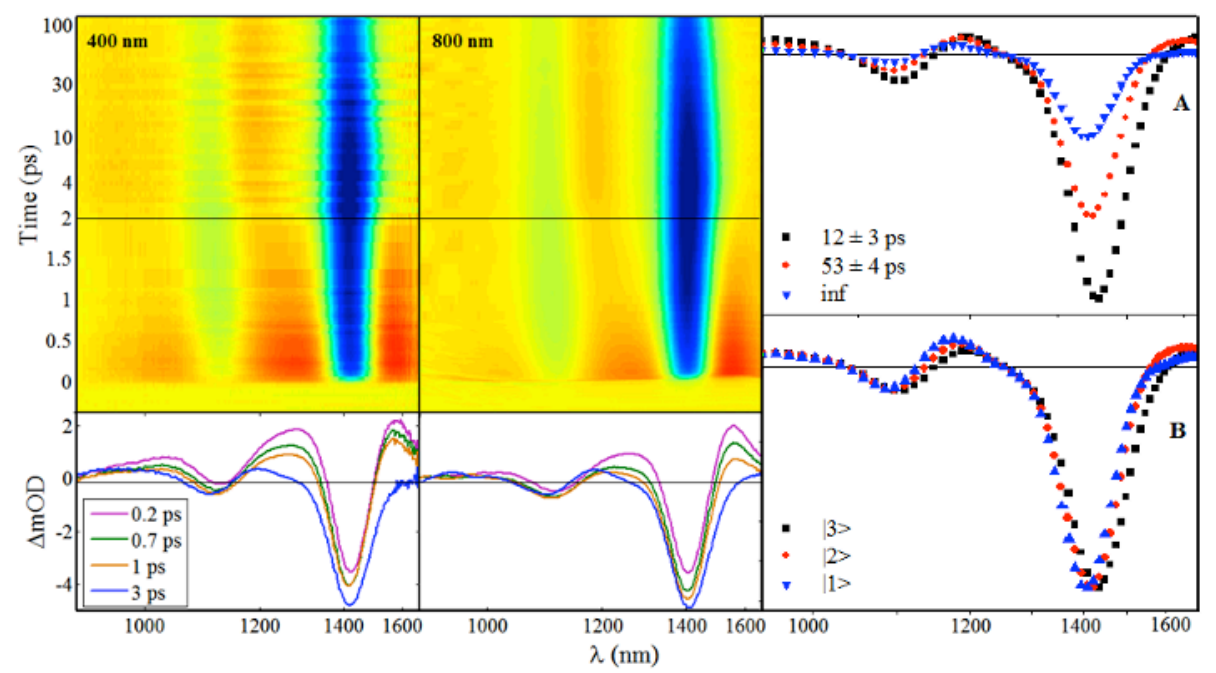

Fig. 1. Transient difference spectra presented above as $\triangle \mathrm{OD}$ maps, below as temporal cuts at the designated pump-probe delays, after excitation at 800 and $400 \mathrm{~nm}$. SADS along with associated lifetimes after excitation at $800 \mathrm{~nm}(\mathrm{~A})$ and normalized to the number of excitons (B) are presented in the right column

\subsection{Global kinetic analysis}

The In an effort to separate the contributions to the transient spectra of various multi-exciton states, $800 \mathrm{~nm}$ pump data was globaly fitted to a sequential kinetic scheme. The distribution of the initial exciton follows the Poisson distribution and was calculated accordingly. Results of this analysis are presented in the right panel of figure 1 in the form of species associated difference spectra (SADS).

The analysis show that a) the band edge bleach peak red shifts progressively, and its amplitude scales nearly perfectly linearly with number of excitons $(\mathrm{N})$. b) the second peak, assigned to the 
1P1P is only shifted to the red due to bi-excitonic interactions. A quantitative determination of this binding interaction gives a value of $16 \pm 2 \mathrm{meV}$ and appear to be linear up to $\mathrm{N}=3$.

\subsection{Testing for existence of MEG}

Figure 2 depicts a comparison of the spectral evolution (left) and kinetics of the band edge (right), throughout the decay of the excitons. As in the previous study using this approach [4], a single scaling factor, related to the ratio of the samples absorption cross sections at both excitation wavelengths, brings the spectral and temporal cuts into perfect overlap. In particular, no excess in bleach was observed at early times after $400 \mathrm{~nm}$ excitation, which would have been indicative of the presence of MEG.

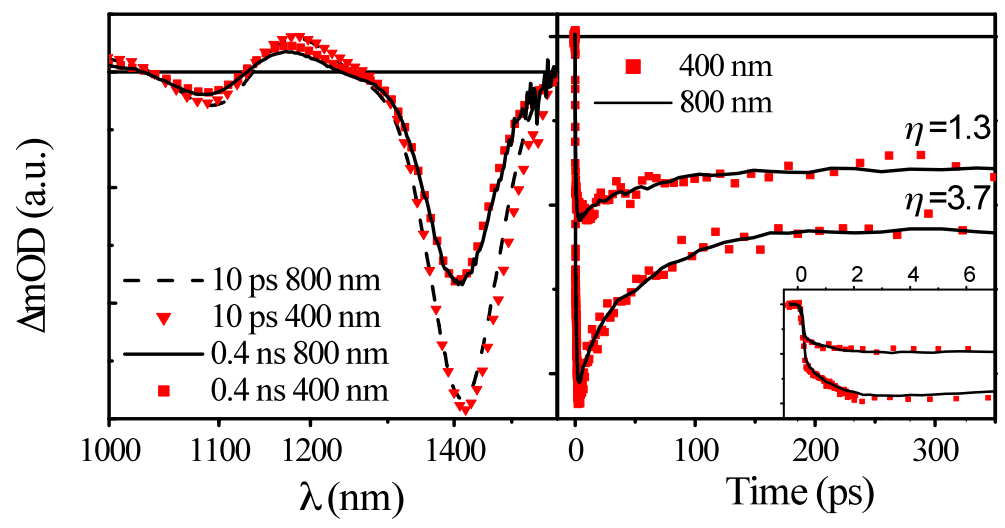

Fig. 2. Comparison of equivalent experiments with 800 and $400 \mathrm{~nm}$ excitation. Left - transient difference spectra taken at the designated pump-probe delays at two pump fluences. Right - comparison of spectral cuts in the data centered at the band-edge absorption feature following $400 \mathrm{~nm}$ (squares) and $800 \mathrm{~nm}$ (solid lines). Insets detail the initial $6 \mathrm{ps}$ of the data.

\section{Conclusions}

Hyperspectral near IR probing provides broad-band spectral coverage of hot exciton relaxation and recombination. The optical generation of nascent high energy excitons leads at early times to a strong excess absorption covering the first $\sim 0.5 \mathrm{eV}$ above the band edge, superimposed with additional fine structure associated with the localized transitions observed in the linear absorption spectrum. Following the cooling, separation of single, double and even triple exciton state contributions to these spectra agree with recent assignments to state filling and bi-exciton binding effects. In the case of the alleged $1 \mathrm{p}-1 \mathrm{p}$ excited transition, the observed shifts are linear with exciton number and $16 \mathrm{meV}$ in amplitude. In addition a recently devised approach which allows direct comparison between equivalent experiments at different pump photon energies to detect the existence of multi-exciton generation (MEG) was employed on a $0.87 \mathrm{eV}$ bang gap lead selenide nanocrystal sample. No sign of MEG was observed up to a photon energy of $3.2 \mathrm{eV}$.

1. R. J. Ellingson, M. C. Beard, J. C. Johnson, P. Yu, O. I. Micic, A. J. Nozik, A. Shabaev, A. L. Efros, Nano Lett. 5, 865 (2005).

2. R. D Schaller. V. I. Klimov, Phys. Rev. Lett 92, 186601 (2004).

3. G. Nair, S.M. Geyer, L. Chang, M. G. Bawendi, Phy Rev B 78, 125325 (2008).

4. M. Ben-Lulu, D. Mocatta, M. Bonn, U. Banin, S. Ruhman, Nano Lett. 8, 1207 (2008).

5. M. Tuan Trinh, A. J. Houtepen, J. M. Schins, J. Piris, L. D. A. Siebbeles. Nano Lett. 8, 2112 (2008). 